\title{
$X$-band active-passive rf pulse compressor with plasma switches
}

\author{
A. L. Vikharev, ${ }^{1,2}$ O. A. Ivanov, ${ }^{1,2}$ A. M. Gorbachev, ${ }^{1,2}$ M. A. Lobaev, ${ }^{1}$ V. A. Isaev, ${ }^{1}$ S. G. Tantawi, ${ }^{3}$ \\ J. R. Lewandowski, ${ }^{3}$ and J. L. Hirshfield ${ }^{2,4}$ \\ ${ }^{1}$ Institute of Applied Physics RAS, Nizhny Novgorod, 603600, Russia \\ ${ }^{2}$ Omega-P, Inc., New Haven, Connecticut 06510, USA \\ ${ }^{3}$ SLAC National Accelerator Laboratory, Menlo Park, California 94025, USA \\ ${ }^{4}$ Department of Physics, Yale University, New Haven, Connecticut 06511, USA
}

(Received 5 September 2011; published 8 December 2011)

\begin{abstract}
As proposed by SLAC, the efficiency of a pulse compressor of the SLED-II type could be increased by changing both the phase of the microwave source and the coupling coefficient of the delay line. In the existing SLED-II system at frequency $11.4 \mathrm{GHz}$, the resonant delay line is coupled with the source via an iris with a constant reflection coefficient. Replacement of the iris with an active component makes it possible to create an active SLED-II system. In this paper, the use of plasma switches as the active elements is discussed. Plasma switches have been developed and tested at a high-power level for production of flattop compressed pulses. Active switching of SLED-II has demonstrated a marked increase in efficiency (by 20\%) and power gain (by 37\%) as compared with passive switching. The active compressor has produced $173 \mathrm{~ns}$ rf flattop output pulses with a power of about $112 \mathrm{MW}$.
\end{abstract}

DOI: 10.1103/PhysRevSTAB.14.121302

PACS numbers: $84.40 . \mathrm{Az}$

\section{INTRODUCTION}

The rf pulse compressors are a rather promising direction for development of a source of high-power nanosecond microwaves for use in future electron-positron colliders. As it is known, the SLED-II compression system [1-3] makes it possible to increase the peak microwave power by several times by means of shortening the duration of the klystron pulse. This system is passive, in that all elements in the microwave circuit have time-independent properties. SLED-II consists of a phase shifter acting on the signal from the rf driver, a klystron amplifier, two identical resonant delay lines, and a $3 \mathrm{~dB}$ directional coupler with one arm feeding the accelerating structures. The microwave energy in such a system is stored in lowloss delay lines, using $\mathrm{TE}_{0 n}$ modes of the circular waveguide. The length of each delay line is chosen such as to make the time of round-trip propagation of the wave along the line, $\tau$, equal to the time required by the accelerating structure. The system is shifted from a regime of energy storage into a regime of energy discharge by changing the phase of the klystron output by $180^{\circ}$ during the pulse. Currently, the maximum power achieved at frequency $11.4 \mathrm{GHz}$ by SLED-II amounts to $580 \mathrm{MW}$ for a compression ratio $C=T / \tau=4$ ( $T$ is input-pulse duration) and compressed-pulse duration of $400 \mathrm{~ns}$ [3]. Among the drawbacks of passive systems, for high efficiency one has to operate the system at a relatively low power gain.

Published by the American Physical Society under the terms of the Creative Commons Attribution 3.0 License. Further distribution of this work must maintain attribution to the author(s) and the published article's title, journal citation, and DOI.
Specifically, in experiment [3], the power gain amounted to about three, therefore, to achieve the record-breaking power, four klystrons with a total power of $190 \mathrm{MW}$ were used. It should be noted that the intrinsic efficiency of the SLED-II system decreases very rapidly as compression ratio $C$ increases [2,3]. The efficiency is reduced due to two effects. During the charging phase, some of the energy is reflected and never gets inside the line. Furthermore, after the phase is reversed the energy inside the line is not discharged completely during the compressed-pulse time period.

As proposed by Tantawi, Ruth, and Vlieks [1], the efficiency and power gain of a pulse compressor of the SLED-II type could be increased by changing both the phase of the microwave source and the coupling coefficient of the delay line. In the existing SLED-II system, the resonant delay line is coupled with the source via an iris with a constant reflection coefficient. Replacement of the iris with an active component (plasma switch) makes it possible to create an active SLED-II system. As it was noted in [1,2], between the compression ratios of 6 and 32 the active system has a significant advantage over the passive one. In this case an increase in the coupling of the line in active SLED-II system will reduce the amount of energy left over after the output pulse is finished. This allows more energy to get out of the storage line during the compressed pulse. The estimates showed [1] the power reflection coefficient of the plasma switch for $C \geq 6$ should be decreased from $R_{0}=0.7-0.9$ in the regime of energy storage to $R_{d}=0.1-0.2$ in the regime of energy discharge.

A number of active rf pulse compression systems have been developed and tested at high-power levels using the 
11.4 GHz magnicon [4-6]. In these experiments, several different types of plasma switches were used as the active elements. The best result was demonstrated with a twochannel microwave compressor with plasma switches and combined microwave input/output element [6]. In experiment [6], short 110-cm-long storage cavities and switches with relatively high reflection coefficients $\left(R_{d} \sim 0.6\right)$ were used. Such parameters do not allow one to produce the pulses with rectangular shape. This paper presents the results of studying the operation of the plasma switches with the SLED-II delay lines at a frequency $11.4 \mathrm{GHz}$. The plasma switches have been developed and tested at a highpower level for production of flattop compressed pulses.

\section{PLASMA SWITCH FOR ACTIVE SLED-II SYSTEM}

The schematic diagram and general view of the plasma switch for the active SLED-II compressor are shown in Fig. 1. The plasma switch consists of an electrically controlled reflector 1-3 and an iris 4 (diaphragm). The reflector was designed and built on the basis of a stepwise waveguide widening with a quartz ring-shaped gasdischarge tube in it, as shown in Fig. 1. The reflector has resonance properties. The reflector volume can be adjusted with the aid of a movable short circuit 1 . The discharge tube 3 with a rectangular cross section is milled into a quartz disk, which ensured the fabrication of this part with high precision. When the plasma is produced in the tube, the frequency characteristic of the reflector shifts, and the reflection coefficient of the switch changes.

Let us consider briefly the general concept of operation of the plasma switch. The principle of operation of the switch can be easily understood based on the calculated frequency characteristic shown in Fig. 2. The calculations were made using the finite-difference time-domain method

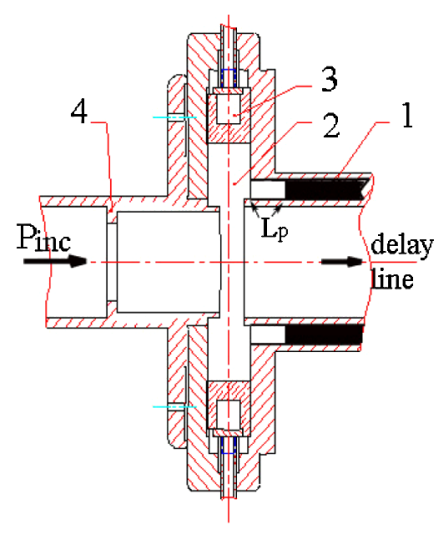

(a)

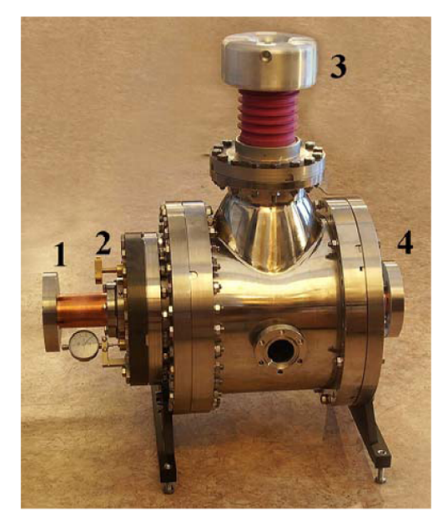

(b)
FIG. 1. (a) Schematic diagram of the plasma switch with reflector and iris (diaphragm): 1-movable short circuit; 2-stepped widening (reflector); 3-gas-discharge tube; 4-iris. (b) General view of plasma switch: 1-input waveguide; 2-adjustment; 3-isolator; 4-output waveguide.
[7] based on directly solving Maxwell's equations in cylindrical coordinates for the rf electric and magnetic fields $\mathrm{E}$ and $\mathrm{H}$. When modeling the reflector switching process, we assumed that plasma with preset electron density is generated in the gas-discharge tube "instantaneously." This assumption is justified, if the characteristic time of plasma formation in the gas-discharge tube is much shorter than the filling time of the reflector $\tau_{R}=Q_{R} / \omega$, where $Q_{R}$ is the loaded $Q$ factor of the reflector and $\omega$ is the frequency of the incident wave. For example, when the reflector $Q$ factor is $Q_{R}=10^{3}$ and the frequency is $f_{0}=11.4 \mathrm{GHz}$, the filling time will be $\tau_{R} \approx 15 \mathrm{~ns}$. The density of the gas and electrons in the quartz tubes were varied in the process of the calculation.

In the regime of energy storage the main part of the incident power $P_{\text {inc }}$ is reflected from the iris at the operating frequency of $11.424 \mathrm{GHz}$. The reflection coefficient $R$ from the reflector is low [curve 1, Fig. 2(a)]. Therefore the reflection coefficient of the iris determines the reflection coefficient of the switch [curve 3, Fig. 2(b)]. When the plasma is produced in the tubes, the frequency characteristic of the reflector is displaced, and the reflection coefficient from it at the operating frequency grows [curve 2, Fig. 2(a)]. In the case of a certain phase incursion between reflector and the iris, the effect of switch brightening occurs and the reflection coefficient drops sharply [curve 4, Fig. 2(b)]. The position of the resonance minimum for the switch with the plasma depends on the plasma density, the geometric dimensions of the reflector, and the distance between the reflector and the iris. Thus, by regulating the distance between the reflector and the iris one can change the position of the minimum and, hence, the reflection coefficient $R_{d}$ in the discharge stage. Experiments [5] showed that the more efficient operation of the plasma switch is achieved when the minimum of reflection coefficients of the switch [curve 4, Fig. 2(b)] is performed at the left-hand branch of the resonance curve of the reflector [curve 2, Fig. 2(b)]. Therefore, the switch for active SLED-II compressor was made with such behavior of frequency characteristics of the reflector and the switch [Fig. 2(b)]. The distribution of the electric field in the switch in the energy storage and in the energy discharge regimes is shown in Fig. 3.

It is seen from the figure that when the plasma appears in the tubes, the switch "brightens up" and the transmission coefficient increases. For input power $P_{\text {inc }}=20 \mathrm{MW}$, the stationary power of microwaves falling on the switch from the side of the resonant delay line reaches $P_{0}=200 \mathrm{MW}$ in the regime of energy storage. In this case, the calculations showed that the amplitude of the electric field in the tubes does not exceed $E_{t}=6.4 \mathrm{kV} / \mathrm{cm}$. Such an electric field is below the level corresponding to the microwave breakdown threshold of the gas filling the tube and surface discharge effects. In the energy discharge stage of the cavity, the high electric strength of the switch is ensured 


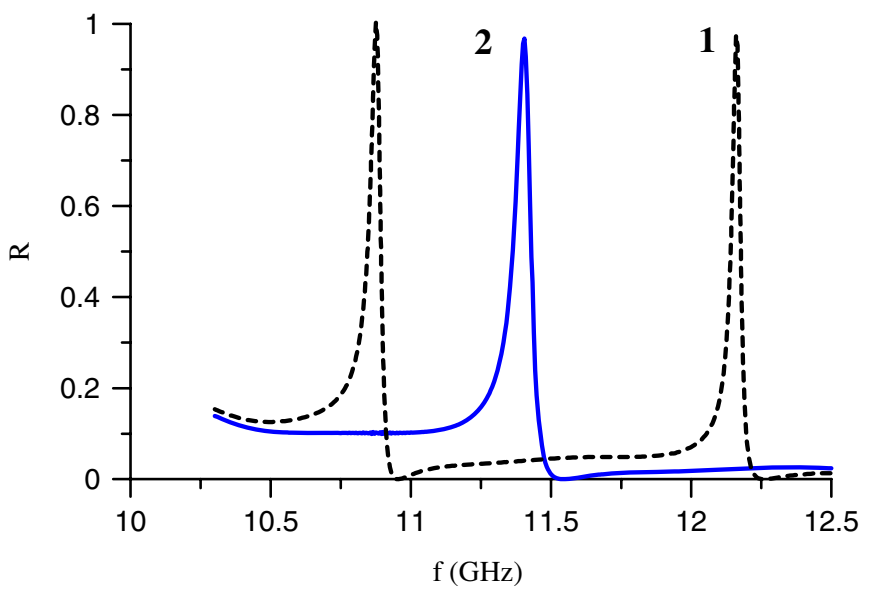

(a)

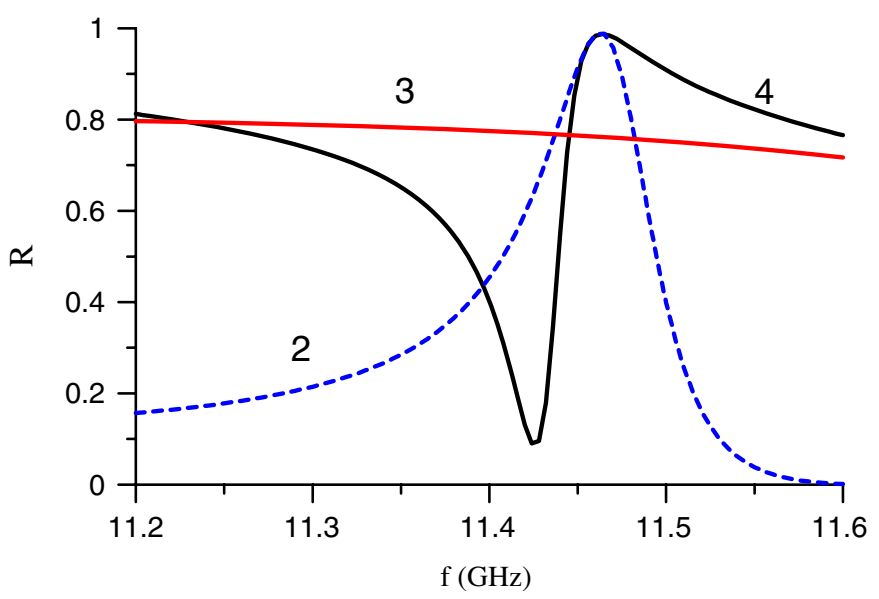

(b)

FIG. 2. Calculated frequency characteristics of the reflector (a) and the switch (b): reflection coefficients of the reflector in the passive state (1), and the switched state (2); reflection coefficients of the switch in the passive state $R_{0}$ (3), and the switched state $R_{d}$ (4).

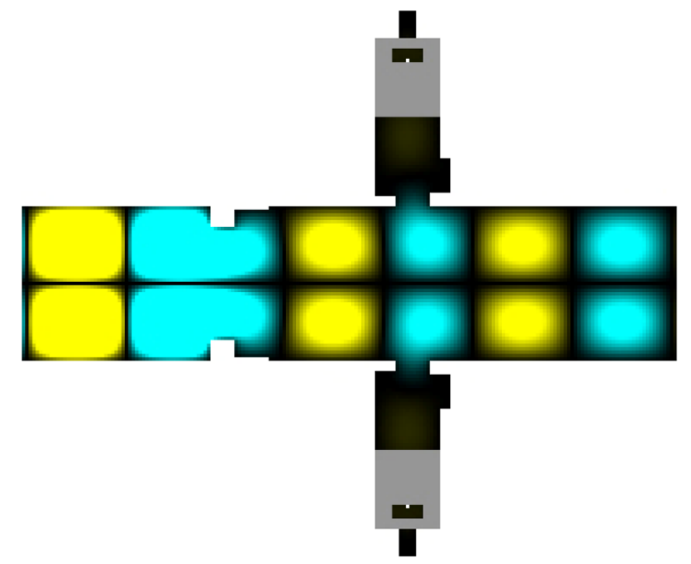

(a)

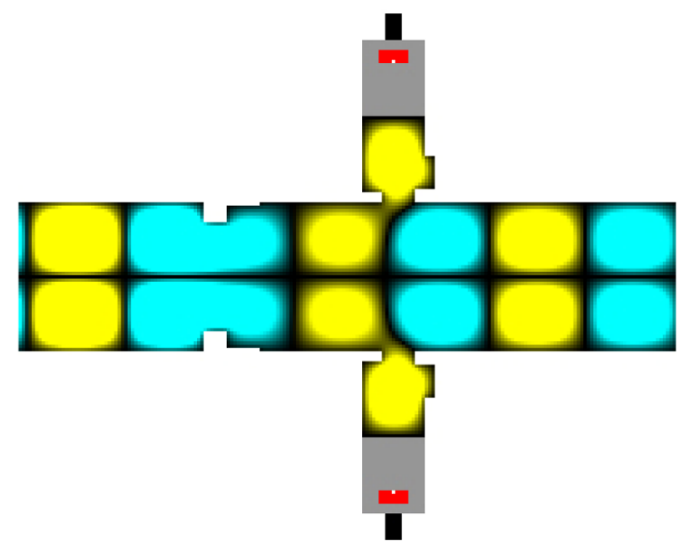

(b)

FIG. 3. Distribution of the electric field in the switch: (a) in the energy storage regime; (b) in the energy discharge regime.

[see Fig. 3(b)] by placing the tube surface in the node of the field of the standing wave excited in the reflector.

Calculations showed that the intensity of the electric field in the switch and the magnitude of electron density at which efficient switching is observed are strongly dependent on the position $L_{p}$ [Fig. 1(a)] of the short circuit. The transmission coefficient in the switched stage $k T=$ $1-R_{d}$ and the maximum electric field inside the gasdischarge tube as functions of the electron density for different positions $L_{p}$ of the adjustment piston are shown in Fig. 4.

It is seen that, for each position of the tuning piston, there is some optimal electron density, at which the switching coefficient reaches a maximum value. The optimal value of the electron density decreases as the piston moves towards the stepwise widening. Really, there is a preset detuning of the resonance frequency of the reflector with respect to the klystron frequency before switching. This detuning decreases when the short moves inside the reflector. When the plasma with a certain (optimal) density is produced in the tubes, the eigenfrequency of the reflector is displaced to the operating frequency and the effect of switch brightening occurs. When this detuning is small, a lower electron density is required to tune the switch into resonance. Thus, one can achieve the optimal switching ratio for a given electron density by changing the position of the piston. It should be noted that the maximum of the electric field is shifted to the left from the maximum of the transmission coefficient $k T$. In this case, when the microwave field causes additional ionization in the tube, the transmission coefficient increases and reaches its maximum. Then, the electron density exceeds the optimal value, and hence, the electric field decreases and electron density is stabilized at a certain level. In this case, the transmission coefficient $k T$ remains relatively high in a wide range of electron densities. 


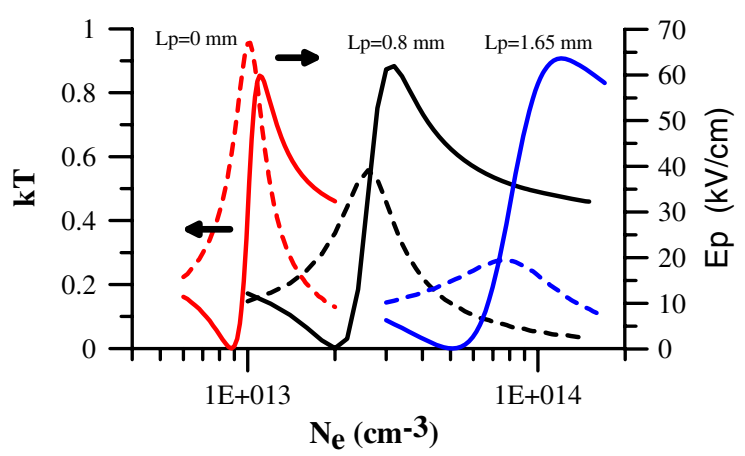

FIG. 4. Dependences of the transmission coefficient and the maximum of electric field amplitude in the plasma on electron density at different position $L_{p}$ of the tuning piston.

\section{THE ACTIVE SLED-II PULSE COMPRESSOR AND THE RESULTS OF THE HIGH POWER TESTS}

Primarily, the switch parameters were optimized to fit the parameters of the SLED-II compression system operating on one $\mathrm{TE}_{01}$ mode of the circular waveguide:

$$
\tau=173 \mathrm{~ns}, \quad T \sim 1.6 \mu \mathrm{s}, \quad C=T / \tau=9,
$$

where $\tau=2 L / V_{g}$ is the round-trip delay through the single moded delay line, $V_{g}$ is the group velocity of the $\mathrm{TE}_{01}$ mode, $L$ is the length of delay line, $T$ is the duration of the input pulse, and $C$ is the compression ratio. The coupling to the lines was optimized for the maximum possible amount of energy storage in the time interval $(C-1) \tau=8 \tau$. The calculated dependence of the efficiency of energy storage in the resonant delay line on the coefficient $R_{0}$ of reflection from the iris is shown in Fig. 5(a). To increase the efficiency of energy storage in the delay lines, the reflection coefficient of the iris was chosen equal to the optimal value $R_{0}=R_{0_{\text {opt }}}=0.717$ (marked with a dot). As a result, the efficiency of energy storage reached $82 \%$. The time dependence of the signal reflected from the SLED-II compressor in the energy storage regime, for this case, is shown in Fig. 5(b). Initially, the line is empty and a large portion of incident power is reflected. Gradually, the reflected power decreases as the line is filled with energy. The reflected power starts to increase again as the line becomes almost fully charged.

According to [2], efficient output of the energy from the storing cavity requires changing the reflection coefficient from $R_{0}$ to $R_{d}$ simultaneously with the phase switching by $180^{\circ}$. The optimal reflection coefficient $R_{d}$ in the calculations was determined from the condition of the absence of the wave reflected from the switch into the cavity at the moment of the phase switching before the last ninth time interval $\tau$. It was assumed then that the round-trip power loss in the storage lines is $\alpha_{\mathrm{ohm}}=3.1 \%$. This number was calculated based on the experimental value of the loaded $Q$ factor of the compressor and the reflection coefficient, $R_{0}$. Simulations showed that, for $R_{0 \text { opt }}=0.717$, the reflection coefficient in the energy discharge regime should be equal to $R_{d}=0.147$ to achieve the maximum power gain.

This reflection coefficient is greater than zero and the switch needs only change the reflection coefficient of the iris between $R_{0_{\text {opt }}}$ and $R_{d}$. In this case, the calculated power gains have the following value:

$$
M_{\mathrm{pas}}=4.54, \quad M_{\mathrm{ac}}=3.4, \quad M_{\mathrm{ac}}=6.9,
$$

where $M_{\text {pas }}$ is the passive power gain due to solely phase switching, $M_{\mathrm{ac}}$ is the power gain due to the switch in the absence of phase switching, and $M_{\mathrm{ac}_{\mathrm{pas}}}$ is the power gain resulting from simultaneously switching the phase and firing the plasma switch.

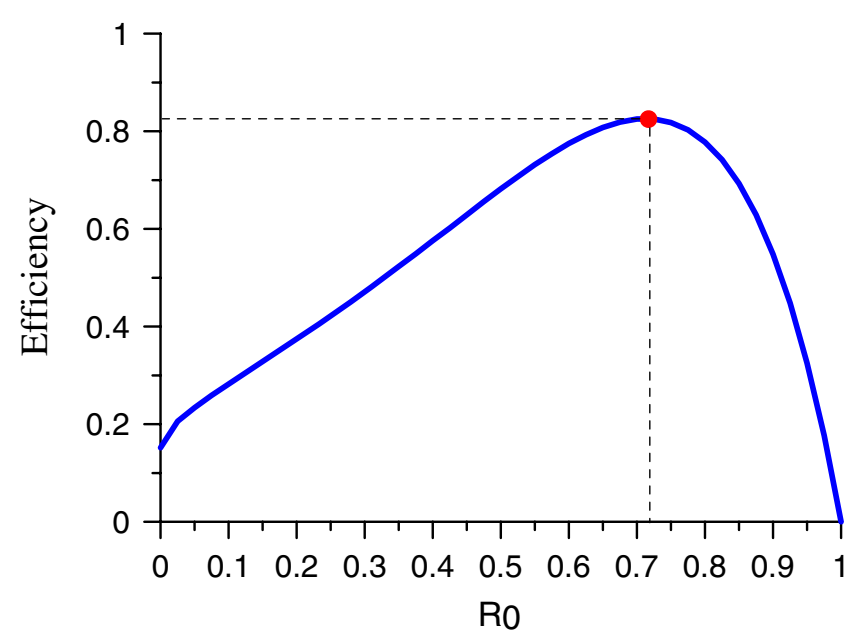

(a)

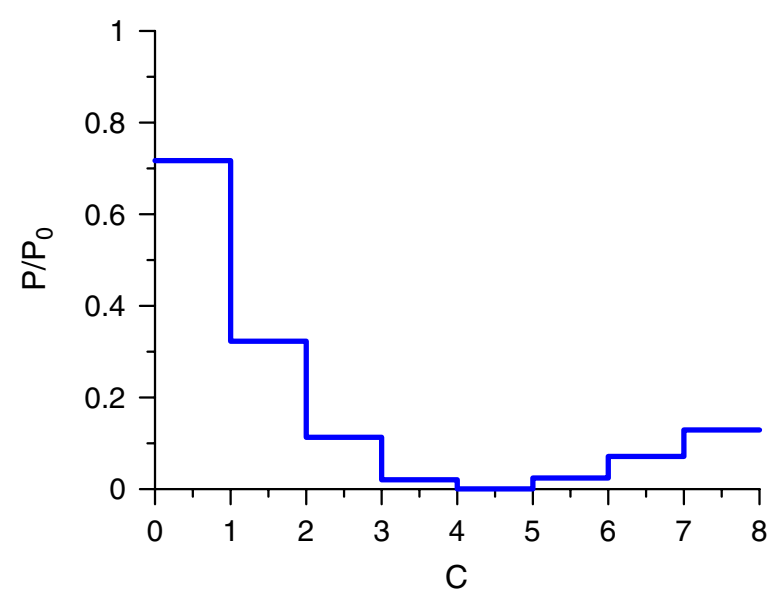

(b)

FIG. 5. (a) Efficiency of energy storage in the resonant delay lines as a function of the reflection coefficient $R_{0}$ of the input iris. (b) The power reflected from the SLED-II compressor in the energy storage regime vs time $\left(t=8 \tau, R_{0}=0.717, P_{0}\right.$ is incident power). 


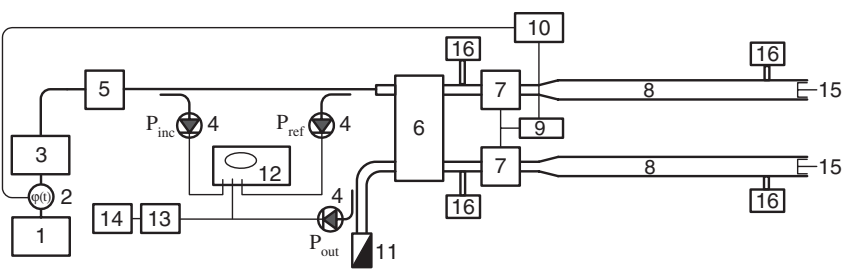

FIG. 6. Schematic diagram of the experimental setup: 1-microwave generator; 2-phase shifter; 3-traveling-wave tube; 4-crystal; 5-klystron; 6-combiner; 7-plasma switch; 8-delay line; 9-high-voltage generator; 10-delay generator; 11-load; 12-oscilloscope; 13-power meter; 14-computer; 15-movable short circuit; 16-ion pump.

At the next stage of the research an active SLED-II system with developed plasma switches was investigated at the high-power level. A schematic diagram of the experimental setup for high-power tests and the general view of the two-channel active SLED-II pulse compressor with plasma switches are shown in Figs. 6 and 7.

Each active SLED-II compressor channel was formed by the plasma switch, a smooth tapered transition, a copper delay line ( $\sim 35 \mathrm{~m}$ long $)$, and a movable short circuit. Two
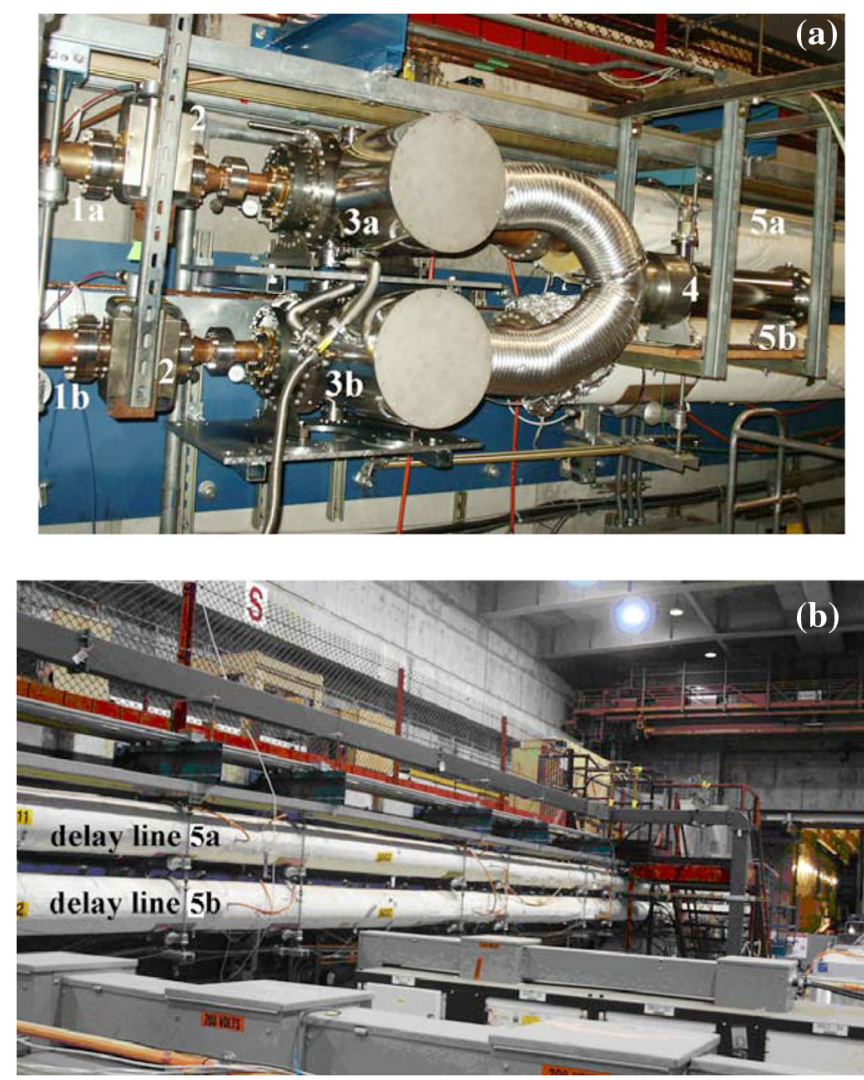

FIG. 7. General view of two-channel active SLED-II pulse compressor: (a) 1a,b- top and lower input waveguides (operating at the mode $\left.\mathrm{TE}_{01}\right) ; 2$-ion pump; 3a,b-top and lower plasma switches; 4-high-voltage generator; 5a,b-top and bottom storage delay line. (b) Top and lower storage delay line. types of gas-discharge tubes were used in the experiment, specifically, one made by using special nonconducting high-temperature glue, and the other made entirely of quartz (unfortunately, we managed to manufacture only one such tube). A glued gas-discharge tube was installed in one of the switches (3a), and in the other (3b) we installed a full-body soldered quartz tube. The resonant delay line was tuned to the operating frequency $f_{0}=11.424 \mathrm{GHz}$ by means of moving the short circuits at the end of the delay lines, and was excited at the $\mathrm{TE}_{01}$ mode of the circular waveguide. The compressor was excited by one klystron at a power level up to $25 \mathrm{MW}$. Signals proportional to the input power $P_{\text {inc }}$ and the output power $P_{\text {out }}$ were measured. The plasma was produced simultaneously in the switches of both channels by one and the same high-voltage pulse generator (pulser). A high-voltage pulse with an amplitude of $60 \mathrm{kV}$ and a duration of $100 \mathrm{~ns}$ was fed to the electrodes of the gas-discharge tube via a $100 \mathrm{Ohm}$ limiting resistor. The klystron modulator and the delay generator (10) were synchronized with the same trigger pulses. From the delay generator the trigger pulse was sent to the phase shifter (2) and pulser (9). This synchronization scheme made it possible to create plasma and change the phase of incident wave simultaneously just before the last time interval $\tau$. The pressure of nitrogen in the gas-discharge tubes was changed in the range $(1-30) \times 10^{-1}$ Torr. The experiment registered the compressed pulses obtained only for the case of passive compression (when the phase of the incident microwave radiation was inverted), and then when both types of the compression (passive and active) were used. The oscillograms of the compressed pulses obtained by using the different types of compression schemes are shown in Fig. 8.

It is seen from the oscillogram (curve 3 ) that the use of a combination of the active and passive compression resulted in a higher efficiency of energy extraction from storage line and a higher amplitudes of the compressed pulse. Really, at the moment when a high-voltage pulse is fed in just before the last time bin, the reflection coefficient decreases sharply and, correspondingly the power, which is transmitted from the compressor to the load, increases. This reduces the amount of energy left over after the output pulse is finished. As a result, the power gain, which is determined as the ratio of the power in the compressed pulse with the duration $\tau$ to the power in the incident pulse, grew by $25 \%-37 \%$. The compression efficiency, which is determined as the ratio of the compressed-pulse energy to the energy in the input pulse before the start of the energy discharge, increased by $9 \%-20 \%$. The compressed pulses obtained in the experiments reached a power level up to $112 \mathrm{MW}$ at a level of incident power, $P_{\text {inc }}$, of $25 \mathrm{MW}$ and duration $\tau=173 \mathrm{~ns}$.

It should be noted that the parameters of the compressed pulses, which were achieved in the experiment, turned out to be somewhat lower than the calculated ones (2). The 


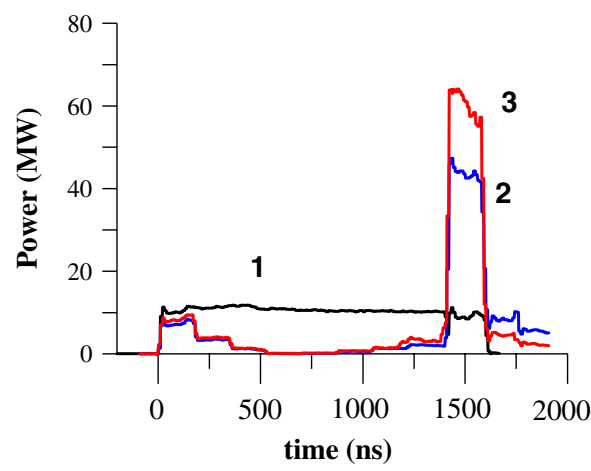

(a)

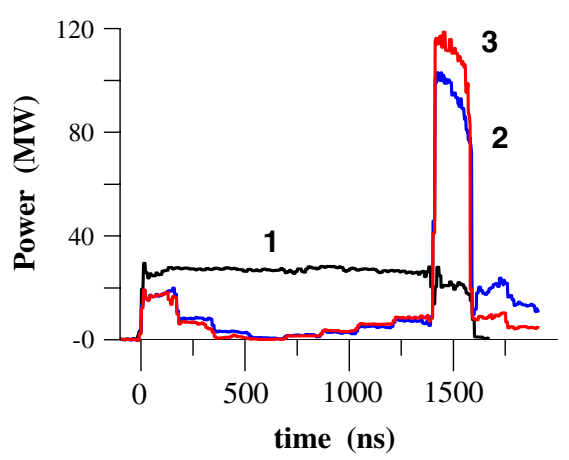

(b)

FIG. 8. Envelope of the compressed pulse of the SLED-II pulse compression system with the plasma switch (pressure of nitrogen 0.3 Torr): 1 -incident pulse; 2-passive compression; 3 -combination of active and passive compression. (a) $P_{\text {inc }}=10.2$ MW, $P_{\text {out }}=58 \mathrm{MW}$, passive power gain 4.2, passive and active power gain 5.8, passive efficiency $52 \%$, passive and active efficiency $72 \%$. (b) $P_{\text {inc }}=25.3 \mathrm{MW}, P_{\text {out }}=112 \mathrm{MW}$, passive power gain 3.6, passive and active power gain 4.5, passive efficiency $45 \%$, passive and active efficiency $54 \%$.

possible reason is that we could not perform the long-term and total conditioning of the compressor before the start of the experiments. As a result, at high power levels we observed microwave breakdowns in the delay lines and near the quartz tubes in the plasma switches (especially near the glued gas-discharge tube). These breakdowns were accompanied by an increase in the pressure in the compressor delay lines. In their turn, the breakdowns can lead to an increase in the round-trip loss in the delay lines and, therefore, a decrease in the power gain and compression efficiency [2].

We believe that those breakdowns were the reasons of the lower power gains compared with the calculated values, which was observed even in the case of purely passive compression, when the incident power was increased. In that case, the shape of the compressed pulse became less rectangular as compared with the pulses obtained during operation in the low power regime [compare Figs. 8(a) and 8(b)]. At the end of the day, the microwave breakdowns led to a failure of vacuum in the glued gas-discharge tube. Note also that the fullquartz tube demonstrated more stable and reliable operation.

\section{CONCLUSION}

A plasma switch has been developed and tested at a high-power level for an active $X$-band SLED-II system for production of flattop compressed pulses. The experiments demonstrated the possibility of realizing the idea from [1] that the efficiency and power gain of a pulse compressor of the SLED-II type could be increased by changing both the phase of the microwave source and the coupling coefficient of the delay line.

Active switching of SLED-II at SLAC has demonstrated a marked increase in efficiency, as compared with passive switching. The maximum increase was by $37 \%$ for the power gain and by $20 \%$ (from $52 \%$ to $72 \%$ ) for the compression efficiency.

For maximum input-pulse power of $25 \mathrm{MW}$ and the duration of $1.6 \mu \mathrm{s}$, we obtained compressed pulses with a power level of $112 \mathrm{MW}$, a duration of $173 \mathrm{~ns}$, and the power gain of 4.5 . In this case, the compression efficiency amounted to $54 \%$.

The stable operation of the SLED-II system with plasma switches requires processing of the system and the fullquartz gas-discharge tubes.

\section{ACKNOWLEDGMENTS}

This work was supported by the U.S. Department of Energy, Office of High Energy Physics.

[1] S. G. Tantawi, R. D. Ruth, and A. E. Vlieks, Nucl. Instrum. Methods Phys. Res., Sect. A 370, 297 (1996).

[2] S. G. Tantawi, R. D. Ruth, A. E. Vlieks, and M. Zolotorev, IEEE Trans. Microwave Theory Tech. 45, 1486 (1997).

[3] S. G. Tantawi, C. D. Nantista, V. A. Dolgashev, C. Pearson, J. Nelson, K. Jobe, J. Chan, K. Fant, J. Frisch, and D. Atkinson, Phys. Rev. ST Accel. Beams 8, 042002 (2005).

[4] A. L. Vikharev, A. M. Gorbachev, O. A. Ivanov, V. A. Isaev, S. V. Kuzikov, B. Z. Movshevich, J. L. Hirshfield, and S.H. Gold, Radiophys. Quantum Electron. 51, 539 (2008).

[5] A. L. Vikharev, A. M. Gorbachev, O. A. Ivanov, V. A. Isaev, V. A. Koldanov, S. V. Kuzikov, J. L. Hirshfield, and S.H. Gold, Radiophys. Quantum Electron. 51, 597 (2008).

[6] A.L. Vikharev, A. M. Gorbachev, O. A. Ivanov, V.A. Isaev, S. V. Kuzikov, M. A. Lobaev, J. L. Hirshfield, S. H. Gold, and A. K. Kinkead, Phys. Rev. ST Accel. Beams 12, 062003 (2009).

[7] A. Taflove, Advances in Computational Electrodynamics. The Finite-Difference Time-Domain Method (Artech, London, 1998), p. 724. 